\title{
Conformational Isomers of Calcineurin Follow Distinct Dissociation Pathways
}

\author{
Basak Kükrer, ${ }^{1,2}$ loana M. Barbu, ${ }^{1,2}$ Jeffrey Copps, ${ }^{3}$ Patrick Hogan, ${ }^{4}$ Susan S. Taylor, ${ }^{3}$ \\ Esther van Duijn, ${ }^{1,2}$ Albert J. R. Heck ${ }^{1,2}$ \\ ${ }^{1}$ Biomolecular Mass Spectrometry and Proteomics, Bijvoet Center for Biomolecular Research and Utrecht Institute \\ for Pharmaceutical Sciences, Utrecht University, Padualaan 8, 3584 CH, Utrecht, The Netherlands \\ ${ }^{2}$ Netherlands Proteomics Centre, Utrecht, The Netherlands \\ ${ }^{3}$ The Howard Hughes Medical Institute, Department of Chemistry and Biochemistry, University of California San Diego, \\ La Jolla, CA, USA \\ ${ }^{4}$ La Jolla Institute for Allergy and Immunology, La Jolla, CA, USA
}

\begin{abstract}
In the gas-phase, ions of protein complexes typically follow an asymmetric dissociation pathway upon collisional activation, whereby an expelled small monomer takes a disproportionately large amount of the charges from the precursor ion. This phenomenon has been rationalized by assuming that upon activation, a single monomer becomes unfolded, thereby attracting charges to its newly exposed basic residues. Here, we report on the atypical gas-phase dissociation of the therapeutically important, heterodimeric calcium/calmodulin-dependent serine/threonine phosphatase calcineurin, using a combination of tandem mass spectrometry, ion mobility mass spectrometry, and computational modeling. Therefore, a hetero-dimeric calcineurin construct (62 kDa), composed of $\mathrm{CNa}(44 \mathrm{kDa}$, a truncation mutant missing the calmodulin binding and auto-inhibitory domains), and CNb (18 kDa), was used. Upon collisional activation, this hetero-dimer follows the commonly observed dissociation behavior, whereby the smaller CNb becomes highly charged and is expelled. Surprisingly, in addition, a second atypical dissociation pathway, whereby the charge partitioning over the two entities is more symmetric is observed. The presence of two gas-phase conformational isomers of calcineurin as revealed by ion mobility mass spectrometry (IM-MS) may explain the co-occurrence of these two dissociation pathways. We reveal the direct relationship between the conformation of the calcineurin precursor ion and its concomitant dissociation pathway and provide insights into the mechanisms underlying this co-occurrence of the typical and atypical fragmentation mechanisms.
\end{abstract}

Key words: Calcineurin, Heterodimer protein complex, Native mass spectrometry ion mobility MS, Protein complex dissociation, Asymmetric charge partitioning

\section{Introduction}

$\mathrm{P}$ rotein phosphorylation is a critical process in regulating cellular events such as signal transduction and cell cycle

Electronic supplementary material The online version of this article (doi:10.1007/s13361-012-0441-8) contains supplementary material, which is available to authorized users.

Correspondence to: Albert J. R. Heck; e-mail: a.j.r.heck@chem.uu.nl control, and is driven by the interplay between protein kinases and phosphatases. Calcineurin $(\mathrm{CN}$, also known as PP2B) is a member of the serine/threonine phosphatase family. Its activity is regulated by $\mathrm{Ca}^{2+}$ and calmodulin and plays a critical role in many calcium-dependent biological processes, for instance T-cell signaling [1-3]. For its activity, $\mathrm{CN}$ interacts with a variety of co-factors, scaffolds, and substrates. Conversely, the phosphatase activity of $\mathrm{CN}$ can be inhibited by immunophilin-immunosuppressant 
agents, such as cyclophilin A and FKBP-FK506 [4-9]. The nuclear factor of activated T-cells (NFAT) is a target for CN. Blocking the phosphatase activity of $\mathrm{CN}$ leads to T-cell suppression $[5,7,10-12]$.

Full-length $\mathrm{CN}$ is a heterodimeric protein $(78 \mathrm{kDa})$ composed of two subunits: a catalytic A subunit (calcineurin $\mathrm{A}, \mathrm{CNa}, 59 \mathrm{kDa}$ ) and a regulatory $\mathrm{B}$ subunit (calcineurin $\mathrm{B}$, $\mathrm{CNb}, 19 \mathrm{kDa}$ ). Wild-type $\mathrm{CNa}$ consists of a globular $\mathrm{N}$ terminal catalytic domain, a helical $\mathrm{CNb}$ binding segment, a calmodulin binding segment and an auto-inhibitory peptide. $\mathrm{CNb}$ has four EF-hand motifs, typical for calcium binding proteins, where four calcium ions can bind [3, 13-15]. The crystal structure of $\mathrm{CN}$ shows that the core structure of $\mathrm{CNa}$ has a $\beta$-sandwich motif containing the enzyme active site. A long 22-residue helix $(\sim 40 \AA)$ of $\mathrm{CNa}$ extends from the surface of the catalytic domain accommodating a binding site for $\mathrm{CNb}[16,17]$. The phosphatase activity of $\mathrm{CN}$ is stimulated by both calcium binding to $\mathrm{CNb}$ and the calciuminduced binding of calmodulin to $\mathrm{CNa}$ [18].

Here we use a native mass spectrometric approach to study the conformational variability of a truncated form of calcineurin (CNt, [62 kDa]), composed of $\mathrm{CNa}(44 \mathrm{kDa})$ that misses the calmodulin binding and auto-inhibitory domains, and full length $\mathrm{CNb}(18 \mathrm{kDa})$. Collision induced dissociation (CID) is used to activate, unfold and dissociate CNt in a controlled and reproducible manner. The shape/size of the ions is monitored using ion mobility mass spectrometry. During activation, which is thought to be a slow heating process, the accumulated internal energy within the complex becomes distributed and may be followed by subunit ejection. The order of sequential subunit dissociation from a protein complex provides an indication about the structural arrangement within the complex [19-22]. During this dissociation process, the leaving subunit gradually unfolds and attracts a disproportionately high amount of charges relative to its mass. This process leads to a charge dissociation pathway commonly known as "asymmetric charge partitioning" [23, 24].

However, investigating the gas-phase dissociation for $\mathrm{CNt}$ we observed a rather unusual dual charge distribution over the formed fragments. In addition to the common asymmetric dissociation pathway, a symmetric charge partitioning mechanism occurred concomitantly. Our data reveal that the dissociation mechanism is determined by the charge state of the precursor complex ion. Lower charge states show a preference for the symmetric charge partitioning pathway, similar to an observation by Pagel et al., showing minimal unfolding of tetrameric transthyretin prior to dissociation, consequently leading to a more evenly distribution of charges over the formed monomer and trimer [25]. Most likely CNt dissociation can follow routes in parallel, due to the presence of two conformational isomers at the start, as shown by IM-MS. The experimentally obtained collisional cross sections $\left(\Omega\right.$ in $\left.\AA^{2}\right)$ of the $\mathrm{CNt}$ precursor ion and the fragment ions were compared with values obtained by molecular modeling. From these analyses we propose a model for the activation and dissociation of CNt whereby the more compact structure primarily follows the unusual symmetric charge partitioning pathway. The second conformer, slightly larger, displays the common protein complex dissociation behavior, whereby the small $\mathrm{CNb}$ subunit fully unfolds and is expelled with a high number of charges.

\section{Experimental}

\section{Materials}

Ammonium acetate (7.5 M solution, Sigma-Aldrich, Sigma), imidazole, and 3-nitrobenzoic acid (3-NBA) (for mass spectrometry, Sigma-Aldrich, Fluka, Zwijndrecht, The Netherlands) were used. The experiments were performed using a truncated form of calcineurin $(\mathrm{CNt})$. Here, the $\mathrm{CNa}$ subunit contained the phosphatase catalytic domain and $\mathrm{CNb}$ binding region, but lacked the calmodulin binding domain and autoinhibitory sequence; $\mathrm{CNb}$ misses amino acids 2-15. Expression and purification was performed as described previously, with only a few exceptions [26, 27]. Bacterial cells were grown at $37^{\circ} \mathrm{C}$ to an $\mathrm{OD}_{600}$ of 0.5 , and induced with isopropyl $\beta$-D-1thiogalactopyranoside (IPTG) for $16-20 \mathrm{~h}$ at $18{ }^{\circ} \mathrm{C}$. The $\mathrm{CNt}$ complex was purified using a Ni-NTA (nickel-nitrolacetic acid) column (Qiagen), after which the his-tag was removed by thrombin. Next, the protein complex was further purified using a size exclusion and Q-coumn, using a Superdex 200 (GE Healthcare) and Q Sepharose (GE Healthcare) column, respectively.

\section{Sample Preparation}

The CNt protein complex $(32.1 \mu \mathrm{M})$ in $20 \mathrm{mM}$ Tris- $\mathrm{HCl}$ buffer including $100 \mathrm{mM} \mathrm{NaCl}$ and $1 \mathrm{mM}$ tris(2carboxyethyl)phosphine (TCEP) at $\mathrm{pH} 7.9$, was buffer exchanged to $150 \mathrm{mM}$ ammonium acetate $\mathrm{pH} 7.9$, using $5 \mathrm{kDa}$ MWCO spin-filter columns (Vivaspin 500; Sartorius Stedim Biotech GmbH, Göttingen, Germany). Mass spectrometric analyses were performed at $\mathrm{CNt}$ concentrations between 2 and $5 \mu \mathrm{M}$. Final concentration of $10 \mathrm{mM}$ 3-nitro benzoic acid (3-NBA) or imidazole was added to the electrospray solution to enhance or reduce charging of the analytes, respectively. $\mathrm{CNt}$ was also sprayed in the presence of $250 \mu \mathrm{M}$ calcium acetate.

\section{Native (Tandem) MS}

One to two $\mu$ aliquots of samples were loaded into goldcoated borosilicate capillaries made in house (using a Sutter P-97 puller [Sutter Instruments Co., Novato, CA, USA] and an Edwards scan coat six-sputter-coater [Edwards Laboratories, Milpitas, CA, USA]). (Tandem) mass spectra were acquired on a Q-Tof2 mass spectrometer (Waters Corp., Manchester, UK), modified for the analysis of macromolecular protein complexes [28, 29]. Typically, a capillary voltage of $1250 \mathrm{~V}$ was used and a sample cone voltage of 
$120 \mathrm{~V}$. The source backing pressure was elevated to approximately 6 mbar in order to promote collisional cooling. Xenon was used as the collision gas at a pressure of $2 \times 10^{-2} \mathrm{mbar}$. The TOF-MS was operated at a pressure of $1.25 \times 10^{-6}$ mbar. Spectra were calibrated using an aqueous cesium iodide solution at a concentration of $25 \mathrm{mg} / \mathrm{mL}$.

\section{Native Ion Mobility MS}

IM-MS measurements were performed on the Synapt G1 HDMS (Waters, Manchester, UK). Voltage and pressure settings were similar to those described for the Q-Tof2. The ion mobility cell was filled with nitrogen at a pressure of 0.65 mbar. A ramped wave height of $7-19 \mathrm{~V}$ and a wave velocity of $200 \mathrm{~m} / \mathrm{s}$ were used. The trap and transfer were operated at $12 \mathrm{~V}$. For collision induced dissociation experiments, the trap was operated as a collision cell. Argon was used as the collision gas at a pressure of $3.0 \times 10^{-2}$ mbar. The collision (trap) voltage was varied between 12 and $130 \mathrm{~V}$. Calibration of the ion mobility cell, essential to calculate collision cross sections, was performed as described previously using ubiquitin, myoglobin and cytochrome c [30]. Processing of MS data was accomplished using MassLynx 4.1 software (Waters Corp.).

\section{Molecular Modeling}

The CCS values of the protein complex structures were calculated using molecular modeling for a modified structure of calcineurin deposited in the Protein Data Bank (PDB: 1AUI) in order to validate the experimental IM-MS data. The quaternary structure was predicted by using the ITASSER server [31, 32]. The geometries of the $\mathrm{CNa}$ and $\mathrm{CNb}$ subunits were extracted from the structure of the modified calcineurin using Pymol (Schrödinger, USA). Pymol was also used to predict the possible unfolded structures of the calcineurin and its dissociation products. Unfolding of the proteins was done manually followed by relaxation. To perform the unfolding of the proteins the sculpting function implemented in Pymol was used. After relaxation the coordinates of the proteins were saved in PDB files. Molecular modeling was performed with MOBCAL (using the projection approximation (PA) algorithms), described in detail elsewhere [33]. To calculate theoretical collision cross sections the projection approximation method was used, that in general is shown to be in best agreement with the experimental data of proteins and their complexes [34-36].

\section{Results}

\section{Native MS and Tandem MS of Calcineurin}

Native nano-ESI on calcineurin $(\mathrm{CNt})$, sprayed from $150 \mathrm{mM}$ aqueous ammonium acetate, $\mathrm{pH} 7.9$, resulted in the detection of the intact hetero-dimer at three distinct charge states; $15+, 14+$, and $13+$, from which a molecular mass of $62,061 \pm 4 \mathrm{Da}$ could be deduced (Figure 1a). To investigate the dissociation behavior of $\mathrm{CNt}$, individual charge states of these precursor ions were selected and subsequently subjected to increasing collisional activation voltages. The acceleration voltage was systematically increased in steps of $5 \mathrm{~V}$. Interestingly, upon dissociation from the $\mathrm{CN}^{15+}$ precursor ion, a dual charge distribution pattern was observed with two distinct maxima in the charge state distributions for both the $\mathrm{CNa}$ and $\mathrm{CNb}$ fragments, centered around $6+$ and $11+$ for $\mathrm{CNa}$ (Figure 1b, blue line), and 9+ and $4+$ for the complementary $\mathrm{CNb}$ species (Figure $1 \mathrm{~b}$, red line). This indicates that in addition to the common asymmetric charge partitioning pathway $(\boldsymbol{A}$ as represented by the $\mathrm{CNa}^{6+}$ and $\mathrm{CNb}^{9+}$ ion pair), an additional dissociation mechanism occurs, resulting in a more symmetric charge partitioning between both subunits $(\boldsymbol{S}$ as represented by the $\mathrm{CNa}^{11+}$ and $\mathrm{CNb}^{4+}$ ion pair). While for very few systems symmetric dissociation has been observed, $\mathrm{CNt}$ is special as in it is a heterodimer. Normally it requires less energy to unfold a smaller protein to a significant extent, in this case $\mathrm{CNb}$. Following this trend the common dissociation mechanism would be asymmetric, whereby a small protein has a high number of charges. For CNt we observe also highly charged $\mathrm{CNa}$ product ions generated via a symmetric dissociation route. Clearly, symmetric dissociation reflects the charge partitioning in relation to the protein subunits mass and not the absolute number of charges that each of them acquire after $\mathrm{CNt}$ dissociation. In Figure $1 \mathrm{c}$, the relative abundances of the $\mathrm{CNa}$ and $\mathrm{CNb}$ product ion pairs are plotted versus the ion kinetic energy in the laboratory frame of reference $\left(E_{l a b}\right.$, equals collision voltage multiplied by ion charge state). Selection and collisional activation of $\mathrm{CNt}^{14+}$ and $\mathrm{CNt}^{13+}$ provided very similar results. We observed that the complex dissociates into its individual subunits via both the asymmetric and symmetric pathways. To investigate the possible effect of the precursor ion's charge on the dissociation behavior, we added imidazole or 3-NBA to the electrospray solution, expanding the envelope to lower charge states with imidazole $(12+-10+)$ and higher charge states with 3 -NBA (to $18+-16+$ )(data not shown). At lower $\mathrm{CNt}$ precursor ion charge states the conventional asymmetric charge partitioning diminished and symmetric charge dissociation became more dominant. The higher charge states, on the other hand, showed both pathways in parallel, with initiation of the symmetric dissociation at slightly lower activation energy.

\section{Unfolding and Dissociation of Calcineurin Monitored by Tandem IM-MS}

It has been well documented that the dissociation behavior of protein complexes may be linked to their three-dimensional structure $[19,22,37,38]$. Consequently, the observation of two dissociation mechanisms for $\mathrm{CNt}$ may imply the existence of two conformational isomers. Native IM-MS 


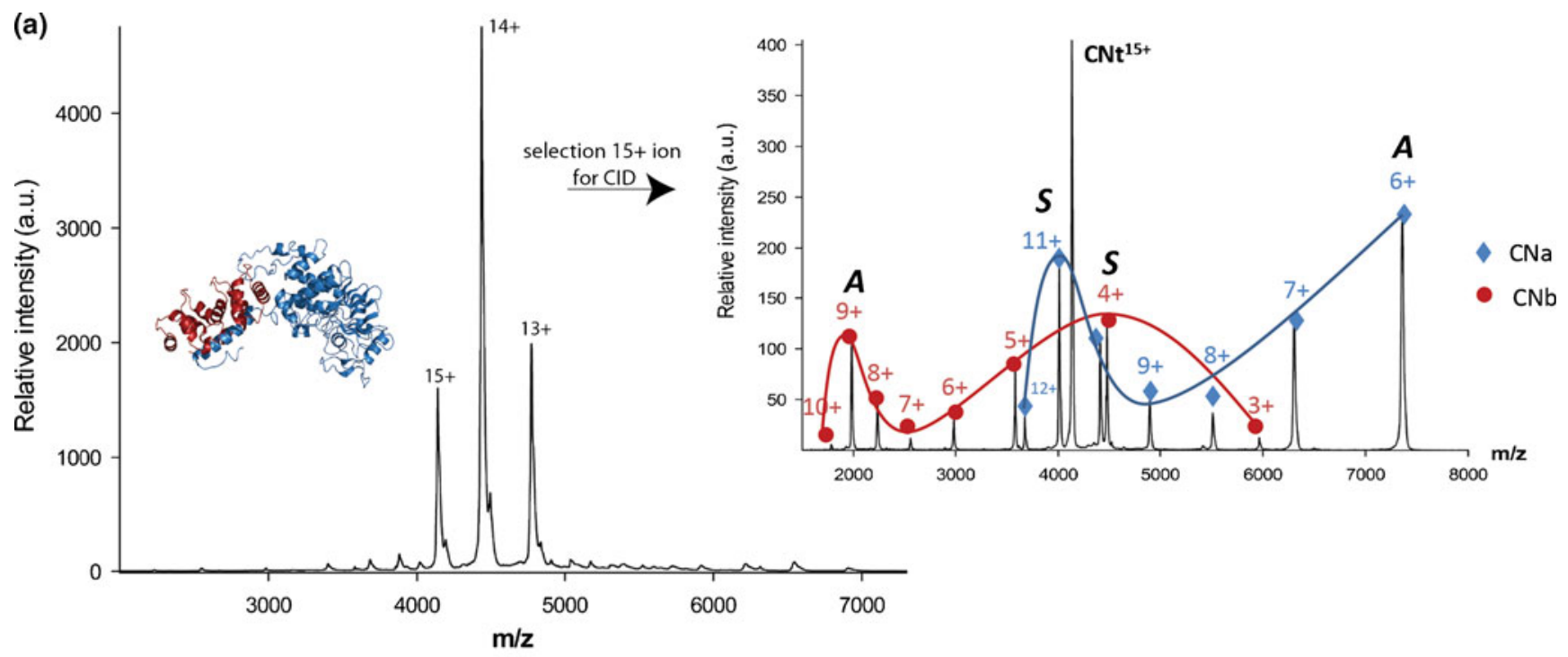

(b)

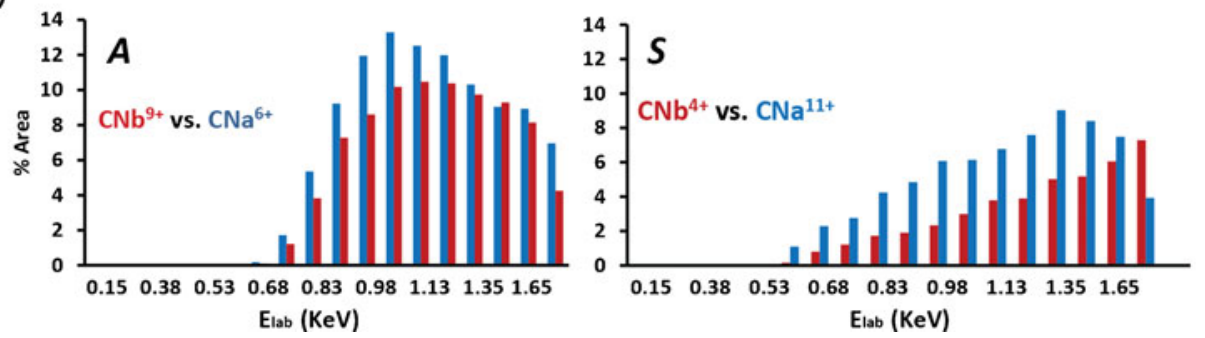

Figure 1. (a) Native nano-ESI mass spectrum of CNt electrosprayed from $150 \mathrm{mM}$ ammonium acetate, $\mathrm{pH} 7.9$, where the most abundant peaks belong to the 15+, 14+, and 13+ charge states of the CNt hetero-dimer. The inset shows the crystal structure of $\mathrm{CNt}$ (CNa subunit in blue, $\mathrm{CNb}$ subunit in red). Inset shows a tandem mass spectrum of $\mathrm{CNt}^{15+}$ at an acceleration voltage of $80 \mathrm{~V}$. Blue diamonds and line: charge states of CNa subunit, red filled circles and line: charge states of CNb subunit. (b) Relative abundances (\%) of ion pair products formed as a function of the ion kinetic energy in the laboratory frame of reference $\left(\mathrm{E}_{\mathrm{lab}}, \mathrm{eV}\right)$. The $\mathrm{CNb}^{9+}-\mathrm{CNa}^{6+}$ ion pair (left) represent an asymmetric charge partitioning $(\boldsymbol{A})$ whereas the symmetric $(\mathbf{S})$ dissociation pathway is illustrated by the $\mathrm{CNb}^{4+}-\mathrm{CNa}^{11+}$ ion pair (right)

experiments indeed confirmed this, as multiple ion mobility drift times for $\mathrm{CNt}$ precursor ions were observed, even at the lowest accelerating voltages. By systematically evaluating the dissociation behavior of various $\mathrm{CNt}$ ions we set out to reveal a possible relation between ion structure and charge state partioning behavior. The conformational changes within $\mathrm{CNt}$ and expelled $\mathrm{CNa}$ and $\mathrm{CNb}$ subunits were monitored by IM-MS as a function of acceleration voltage. In Figure 2, drift time profiles of $\mathrm{CNt}^{15+}, \mathrm{CNa}$ (yellow line), and $\mathrm{CNb}$ (white line) product ions are shown generated at an acceleration voltage of $80 \mathrm{~V}$. A close investigation of the contour plot indicates the existence of multiple conformations for the $\mathrm{CNt}^{15+}$ precursor ion, as well as for some of the product ions; most notably for the $\mathrm{CNb}^{7+}$ and $\mathrm{CNa}^{10+}$ ions.

In Figure 3a, IM-MS drift-time profiles of the $\mathrm{CNt}^{15+}$ precursor ions at increasing acceleration voltages are displayed. The two drift-time maxima at $20 \mathrm{~V}$ for the $\mathrm{CNt}^{15+}$ ions indicate the existence of two different conformational isomers. As the collision energy is increased, the retention times of these components also increase. We can even observe the existence of multiple components at 35 and
$65 \mathrm{~V}$. The calculated $\Omega$ values of the $\mathrm{CNt}^{15+}, \mathrm{CNt}^{14+}$, and $\mathrm{CNt}^{13+}$ precursor ions are summarized in Figure $3 \mathrm{~b}$. Here we plotted the $\Omega$ as a function of $E_{\text {lab }}$, to correct for effects of precursor ion charge. These results clearly show that independent of the selected charge state, the precursor ions exhibit more than one conformational isomer under native mass spectrometric conditions. The two major conformational isomers observed at the lowest activation energy have average $\Omega$ values of approximately 3,650 and $4,000 \AA^{2}$, whereby the relative abundance of the larger structure becomes more dominant with increasing charge.

The reported crystal structure of calcineurin (PDB: 1AUI), allows us to calculate a theoretical $\Omega$ using the molecular modeling program MOBCAL. Here we used the projection approximation (PA) algorithm, resulting in a value of $3533 \AA^{2}$. This is in close agreement with the lower experimental $\Omega$ value of $3650 \AA^{2}$. It is known that for some proteins and protein complexes the PA method underestimates the $\Omega$ [39]. It is possible to apply a linear correction factor to decrease the deviation between theory and experiment [40-42]. However, it also depends on the system how significant the deviation is [34, 


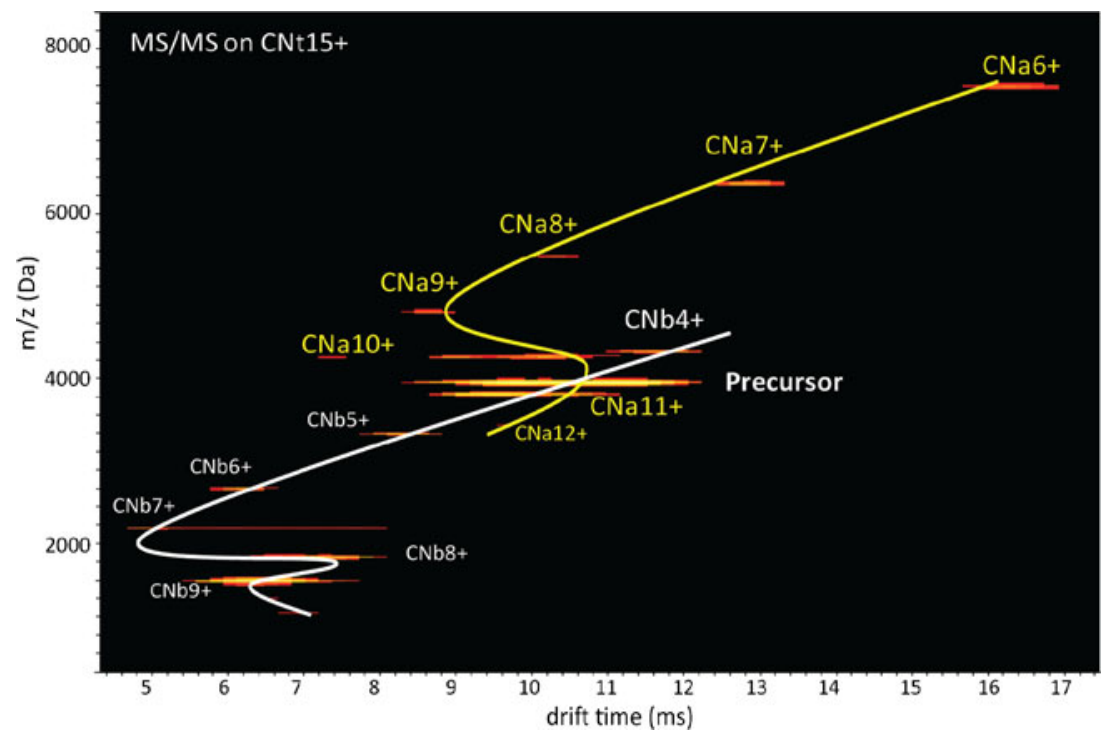

Figure 2. Two-dimensional drift time profile ( $\mathrm{m} / \mathrm{z}$ versus drift time $[\mathrm{ms}])$ of dissociated $\mathrm{CN}^{15+}$, at an acceleration voltage of $80 \mathrm{~V}$ $\left(\mathrm{E}_{\text {lab }}\right.$ of $\left.1200 \mathrm{eV}\right)$. Drift time profiles of the precursor ion and the $\mathrm{CNa}$ and $\mathrm{CNb}$ product ions are displayed. These drift time profiles were used to derive $\Omega$ s for the precursor and product ions

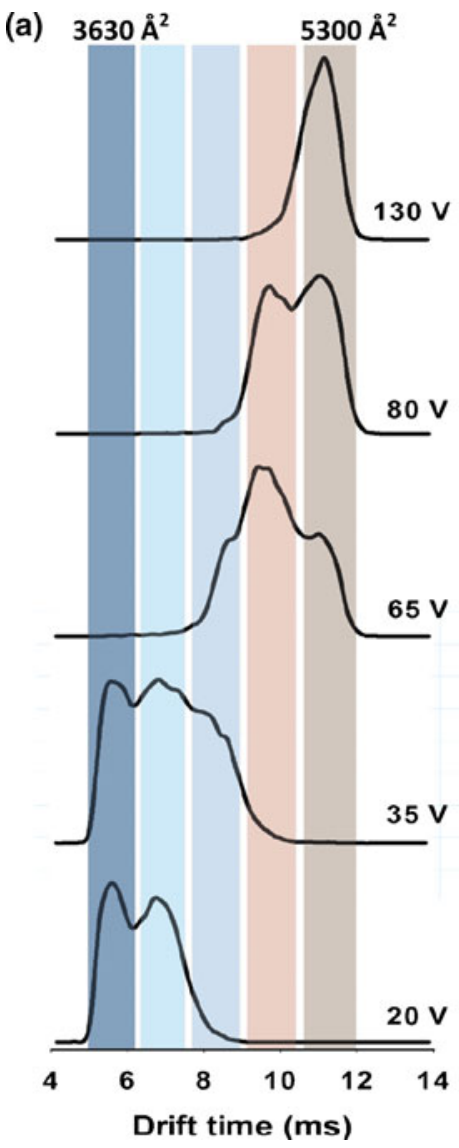

(b)

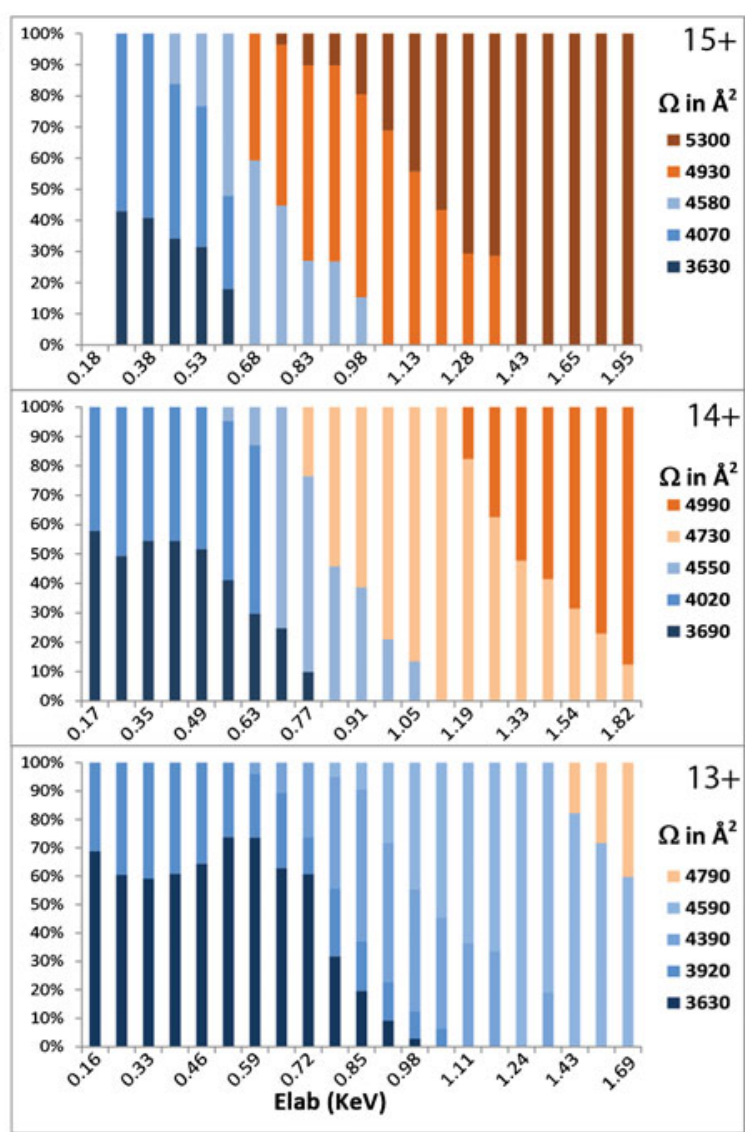

Figure 3. (a) Zoom in of the IM-MS retention profiles of the $\mathrm{CNt}^{15+}$ precursor ion at an acceleration voltage of 20, 35, 65, 80, and $130 \mathrm{~V}$ ( $\mathrm{E}_{\mathrm{lab}}: 300-1950 \mathrm{eV}$ ). Each maximum is interpreted as a different conformational isomer. These retention time maxima are converted into the $\Omega$ s indicated. (b) The relative abundances of the different conformers of the CNt precursor ions (15+, 14+, and $13+)$ as a function of the increasing $E_{\text {lab }}(\mathrm{eV})$. Color bars shown at the right side of the graphs represent different conformational isomers $\left(\Omega\right.$ values, in $\left.\AA^{2}\right)$ 
43]. Here we use only the theoretical $\Omega$ as a guideline to support our experimental data, whereby we focus on trends in increased or decreased $\Omega$ values.

Having calculated a relatively small $\Omega$ for $\mathrm{CNt}$, we hypothesize that the larger structure detected, corresponding to a $\Omega$ of $4000 \AA^{2}$, is due to partial unfolding of the $\mathrm{CNt}$ hetero-dimer. At this moment, we cannot rule out the possibility that the two $\mathrm{CNt}$ conformations originate from a collapsed and native state, instead of a native and unfolded structure. Collapsed protein structures have been observed previously, mainly at higher charge states [35]. As we still observe two distinct conformations at reduced charge states $\left(10^{+}-12^{+}\right.$, not shown), collapsing is less likely to occur and the two CNt structures are expected to represent native and partially unfolded states.

Next, we computationally constructed several metastable states of unfolded CNt (using Pymol), and calculated their $\Omega$. Partial unfolding of the $\mathrm{CNa}$ subunit, when still bound to $\mathrm{CNb}$, results in a $\Omega$ of $4843 \AA^{2}$. Conversely, partially unfolded $\mathrm{CNb}$, still bound to $\mathrm{CNa}$, increased the $\Omega$ to a lesser extent, to $4360 \AA^{2}$. This reveals that under the conditions used, neither $\mathrm{CNa}$ nor $\mathrm{CNb}$ completely unfolds in the intact $\mathrm{CNt}$ complex. To study if these $\mathrm{CNt}$ conformational isomers result in distinct dissociation pathways, we also investigated the dissociation behavior of the lower charge states, whereby we had previously shown that the symmetric charge portioning pathway was dominating. IM-MS performed on $\mathrm{CNt}^{10^{+}}$precursor ion displayed a $\Omega$ of $3440 \AA^{2}$, within $3 \%$ of the $\Omega$ calculated from the crystal structure. Considering the fact that at lower charge states the smaller native-like $\mathrm{CNt}$ structure is more abundant, we take this as an argument that the more native conformer of $\mathrm{CNt}$ prefers to dissociate via the less common symmetric charge partitioning mechanism.

In Figure 4 the experimentally determined $\Omega$ s of all the fragment ions, formed by CID on the $\mathrm{CNt}^{15+}$ precursor ions are summarized. The theoretically calculated $\Omega \mathrm{s}$ of the collapsed and native-like and unfolded $\mathrm{CNa}$ and $\mathrm{CNb}$, are indicated as blue and red bars. $\mathrm{CNa}$ and $\mathrm{CNb}$ were extracted individually from the published crystal structure to calculate their native $\Omega$ using the molecular modeling program MOBCAL. Subsequently unfolded structures of the extracted native-like structures were generated using Pymol. The structures of both monomers were unfolded till a certain extent (at those positions most likely to lose their three dimensional conformation first, e.g., extended helices, flexible loops). Afterwards, these new structures were equilibrated in such a way to generate the lowest energy conformations for the particular protein states of $\mathrm{CNa}$ and $\mathrm{CNb}$.

The experimental $\Omega$ s of the $\mathrm{CNa}$ and $\mathrm{CNb}$ product ions display a similar behavior with increasing charge. In both cases, the collapsed and native-like protein structures are favored when they are formed at lower charge states. The more compact structure of $\mathrm{CNa}$ (experimental value $2565 \AA^{2}$ versus $2413 \AA^{2}$ calculated) may be due to the collapse of the helix that extends from $\mathrm{CNa}$, involved in $\mathrm{CNb}$ binding, in the gas-phase. In the intact $\mathrm{CNt}$ complex a helix is extending from the surface of $\mathrm{CNa}$ that interacts with $\mathrm{CNb}$. Upon $\mathrm{CNb}$ removal the helix becomes an exposed and flexible part of the structure that can easily change its position towards the body of $\mathrm{CNa}$. In case of $\mathrm{CNb}$, that can undergo less dramatic structural rearrangements, a slightly collapsed isoform was modeled $\left(1,427 \AA^{2}\right)$ which is in good agreement with the experimental value $\left(1,435 \AA^{2}\right)$ as given in Figure 5 b. The larger (more unfolded) conformations are observed at higher charge states. Interestingly, our data indicate that the $\mathrm{CNb}^{7+}$ charge state represents a distinct transition state between the native-like structures at lower charge states and unfolded conformations at higher charge states (Figure $4 b$ ).

Beside the $\Omega$ s observed at $80 \mathrm{~V}$ acceleration voltage displayed in Figure 5, we monitored the $\Omega$ s of the product ions as a function of accelerating voltage used for CID In supplementary Figure 1 this is shown for two series of concomitant product ions, representing the symmetric and asymmetric dissociation pathway, respectively. The $\mathrm{CNa}^{11+}$ / $\mathrm{CNb}^{4+}$ concomitant ion pair follows the symmetric chargepartitioning route, clearly, upon dissociation, the larger subunit $\mathrm{CNa}$ becomes significantly unfolded, while almost no structural change is observed for $\mathrm{CNb}$. The opposite is observed for the $\mathrm{CNa}^{6+} / \mathrm{CNb}^{9+}$ ion pair, formed via the asymmetric charge dissociation pathway, whereby the $\mathrm{CNa}$ structure collapses slightly and the $\mathrm{CNb}$ becomes largely unfolded.

\section{Discussion and Conclusions}

The progression of mass spectrometry in the structural biology field is clearly demonstrated by the increasing number of applications, whereby the technique is used to probe inherent details on protein complex composition, conformation and topology [22, 34, 35, 38, 40, 43]. Especially, tandem mass spectrometry and ion mobility mass spectrometry are used more and more. Over the years, it became apparent that independent of the system studied, protein complexes seemed to display a generic gas-phase dissociation pathway. The assembly is energetically activated, and prior to the release of a single subunit, this subunit (partly) unfolds and attracts a disproportionately large amount of charges of the precursor ion, leaving a modestly charged stripped protein(complex) [23, 37, 44-46]. Although, the majority of all studied protein complexes follow this common trend, more recently it has been demonstrated that this dissociation behavior is not fully generic [29, 4749]. Charge partitioning is dependent on the ion's charge state, the conformational flexibility and location of a subunit in a larger protein complex, and interaction area with the other complex components [50,51]. Williams and coworkers have performed detailed studies to decipher the origin of charge partitioning [45, 52, 53]. For example, $\mapsto-$ lactalbumin homodimers, of which both monomers are constrained with intramolecular cross-links primarily disso- 

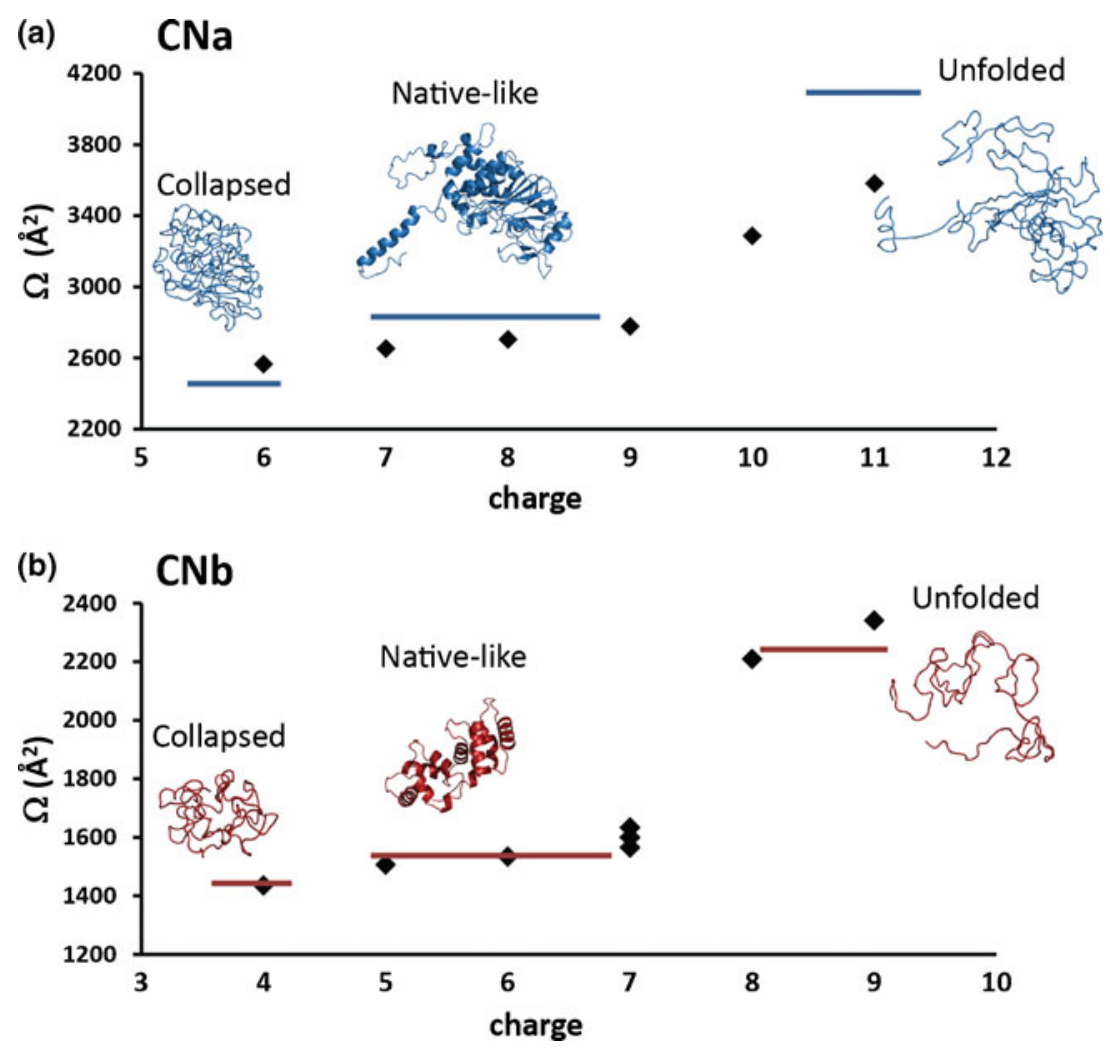

Figure 4. Experimental $\Omega \mathrm{S}$ (diamonds) and $\Omega \mathrm{S}$ calculated from structural models of the individual $\mathrm{CNa}$ (a) and $\mathrm{CNb}$ (b) product ions as a function of their charge. The product ions were formed by performing $\mathrm{CID}$ on the $\mathrm{CNt}^{15+}$ precursor ions at a collision voltage of $80 \mathrm{~V}\left(E_{\text {lab }} 1200 \mathrm{eV}\right)$. The modeled structures of the collapsed, native-like and fully unfolded product ions are displayed along with their modeled $\Omega \mathrm{s}$ (blue and red bars)

(a)
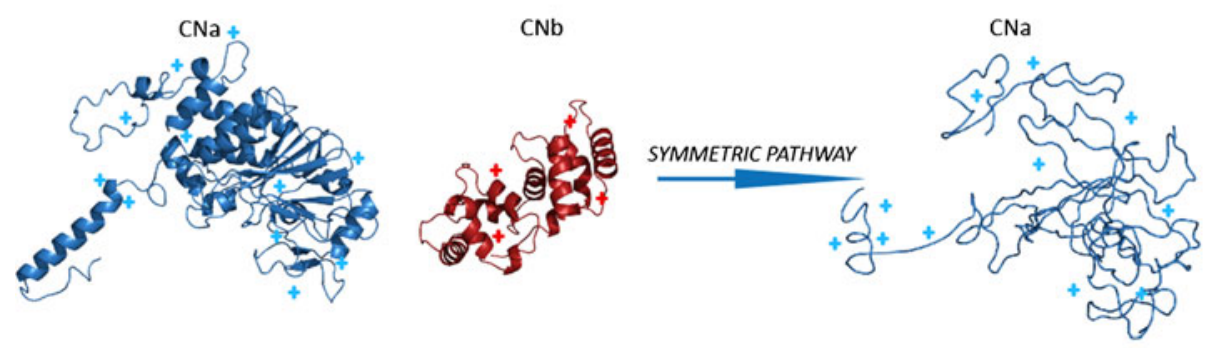

(b)
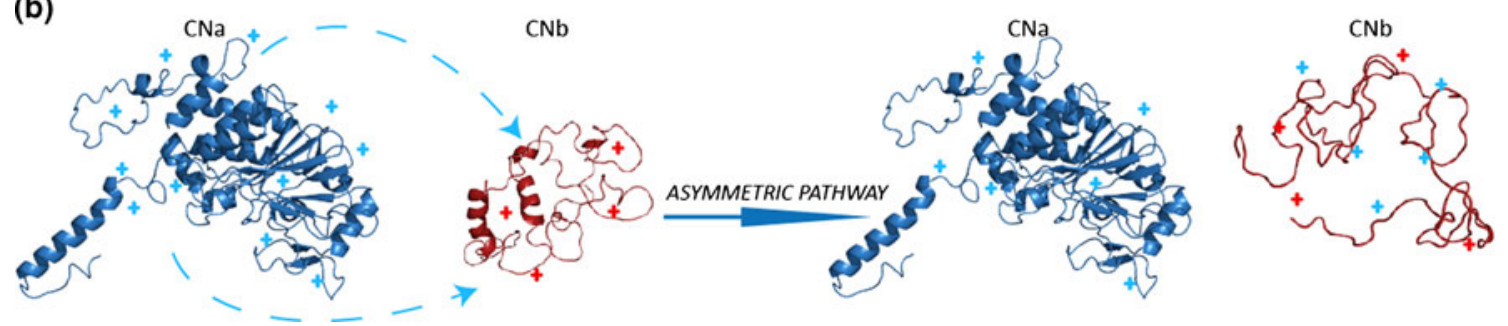

Figure 5. Mechanistic scheme representing the collision induced charge partitioning and dissociation pathways of the CNt heterodimer. Blue and red represent $\mathrm{CNa}$ and $\mathrm{CNb}$ subunits, respectively. For clarity, their structures are represented as individual components also prior to dissociation. (a) Symmetric charge partitioning pathway. Preceding activation CNa and $\mathrm{CNb}$ both remain in their native structure. During dissociation $\mathrm{CNa}$ partially unfolds while the structure of $\mathrm{CNb}$ is largely unaffected. In this scenario, there is insignificant charge relocation and thus after dissociation the charges are separated equally according to the mass of the individual subunits. (b) Asymmetric dissociation pathway. CNa remains largely in its native structure, while $\mathrm{CNb}$ adopts an extended conformation whereby charges are relocated from the $\mathrm{CNa}$ to the $\mathrm{CNb}$ subunit. After collisional activation $\mathrm{CNb}$ is fully unfolded and highly charged. The $\mathrm{CNa}$ fragment retains its more native like conformation 
ciate via the symmetric charge partitioning pathway, while the asymmetric route is favored when the monomers are not cross-linked. Clearly, for this homodimer the origin of asymmetric charge partitioning is due to the unfolding event of a single $\mapsto$-lactalbumin monomer just before the actual dissociation event occurs [45]. However, it is still of particular interest to characterize in more detail what drives certain protein complexes to dissociate via a different route.

Here, we studied CNt, which represents an unusual protein complex system, as it can dissociate via both the symmetric and asymmetric pathway. $\mathrm{CNt}$ is a heterodimer, composed of a large $\mathrm{CNa}(44 \mathrm{kDa})$ and small $\mathrm{CNb}(18 \mathrm{kDa})$ subunit. Therefore, after collisional activation, the formation of highly charged $\mathrm{CNa}$ product ions was unexpected. However, upon dissociation of $\mathrm{CNt}$, both $\mathrm{CNa}$ and $\mathrm{CNb}$ fragments ions generated were centered around two different maxima. We found this to be the result of two conformational $\mathrm{CNt}$ isomers that are slightly different from each other, a compact native like structure and a slightly unfolded CNt complex. Our tandem and IM-MS data indicate that the compact structure follows the unusual symmetric dissociation pathway, while the extended $\mathrm{CNt}$ conformation dissociates via the typical asymmetric charge partitioning route (Figure 5).

Here we postulate two different hypotheses for this unusual behavior of CNt. First, the different $\mathrm{CNt}$ conformations observed might correspond to known physiologic conformations of calcineurin that depend on $\mathrm{Ca}^{2+}$ binding to $\mathrm{CNb}$. As mentioned previously, $\mathrm{CNb}$ contains four calcium binding sites (EF-hand motifs), two with high affinity and two with moderate affinity. Heterogeneity in binding of $\mathrm{Ca}^{2+}$ to these sites may induce different conformational states of $\mathrm{CNb}$ [54]. At the low level of $\mathrm{Ca}^{2+}$ used in the native MS buffer (no additional $\mathrm{Ca}^{2+}$ was added to the spray solution), it is likely that there is incomplete $\mathrm{Ca}^{2+}$ binding.
Partially unfolded $\mathrm{CNb}$, due to insufficient $\mathrm{Ca}^{2+}$ binding would be consistent with a larger cross-section and dissociation by the asymmetric route. The more compact and stabilized form of $\mathrm{CNb}$ might then, in turn, favor the symmetric dissociation pathway. In the calcineurin crystal structure, $\mathrm{CNb}$ is in a compact form, which is stabilized by $\mathrm{Ca}^{2+}$ binding to all four EF-hands and by interaction of all four EF-hands with the $\mathrm{CNb}$-binding helix of $\mathrm{CNa}$. There are two ways that a fraction of $\mathrm{CNt}$ might be stabilized in this conformation. First, at the low level of $\mathrm{Ca}^{2+}$ that is still present in the electrospray buffer, the structural $\mathrm{Ca}^{2+}$ binding sites in the C-terminal half of $\mathrm{CNb}$ will be occupied by $\mathrm{Ca}^{2+}$, but at least a fraction of the loweraffinity $\mathrm{Ca}^{2+}$ binding sites in the first two EF-hands will be unoccupied [18]. Second, since the $\mathrm{CNt}$ dimer lacks a $\mathrm{CNa}$ regulatory domain that would compete with the $\mathrm{N}$-terminal part of $\mathrm{CNb}$ [54], an appreciable fraction of $\mathrm{CNb}$ might assume the conformation seen in the crystal structure, even in the absence of bound $\mathrm{Ca}^{2+}$. In general, our mass spectrometry data confirm this hypothesis, as we do observe differences in $\mathrm{Ca}^{2+}$ binding for the various $\mathrm{CNb}$ ions formed after $\mathrm{CNt}$ dissociation. The $\mathrm{CNb}$ ions formed via the symmetric route occupy a higher number of $\mathrm{Ca}^{2+}$ ions compared with the high charged $\mathrm{CNb}$ ions formed via the usual asymmetric dissociation pathway (Figure 6). However, none of the ions show the full occupancy of the four EF-hand motifs by $\mathrm{Ca}^{2+}$, instead we observe that native like $\mathrm{CNb}$ ions primarily have two $\mathrm{Ca}^{2+}$ ions bound while for the highly charged unfolded $\mathrm{CNb}$ ions also a significant number of ions shows the binding of only one or even no $\mathrm{Ca}^{2+}$ at all. The addition of extra calcium (as an acetate salt) to the spray solution dramatically decreased the quality of our spectra, in terms of ion intensity and resolution. Furthermore, it did not result in the full occupancy of all four sites on $\mathrm{CNb}$.

Our second explanation is more focused on $\mathrm{CNa}$ instead of $\mathrm{CNb}$. Within intact $\mathrm{CNt}$ an obvious characteristic of $\mathrm{CNa}$

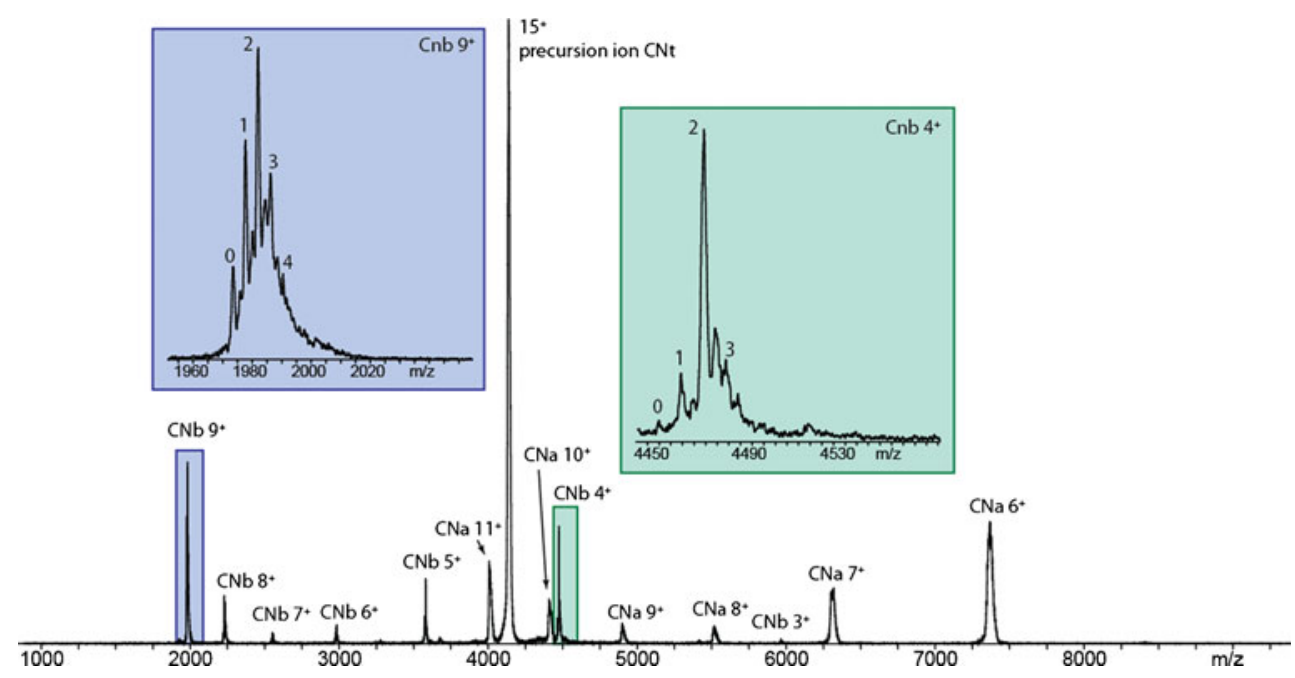

Figure 6. Calcium occupancy on the various $\mathrm{CNb}$ forms formed after collision induced dissociation of $\mathrm{CNt}^{1} 15^{+}$ion. The symmetric charge partitioning route results in the formation of $\mathrm{CNb}^{4+}$ (purple box), whereby $\mathrm{CNb}$ is expected to retain a more native conformation. On the contrary, the $\mathrm{CNb}^{9+}$ ions (green box), originating from the asymmetric dissociation pathway, are more unfolded. In line with this hypothesis the number of calcium ions bound to $\mathrm{CNb}$ is significantly lower for these highly charged $\mathrm{CNb}$ ions. The intermediate peaks not assigned in both insets originate from $\mathrm{Na}^{+}$adducts 
is its extending helix. This non-globular protein conformation could be a possible explanation for the symmetric charge partitioning pathway to occur upon $\mathrm{CNt}$ dissociation. Normally, the smallest subunit unfolds and thereby increases its surface area; simultaneously the unfolding subunit gathers the majority of the ion's charges. Under native like conditions the $\mathrm{CNa}$ adopts a more extended structure, due to the helix pointing away from the rest of the body of the protein, whereby more chargeable residues become accessible for protonation. Upon $\mathrm{CNt}$ activation and the disruption of the noncovalent interactions between $\mathrm{CNa}$ and $\mathrm{CNb}$, charge migration occurs towards the direction of $\mathrm{CNa}$ that then further unfolds, eventually leading to its dissociation via the symmetric pathway. Interestingly, the number of charges are evenly shared among $\mathrm{CNa}$ and $\mathrm{CNb}$, related to their mass, after symmetric charge partitioning. The maxima of the charge state distributions for both fragment ions lies between 4000 and $4500 \mathrm{~m} / z$. Still, these "relatively" highly charged $\mathrm{CNa}$ ions are not fully unfolded, as parts of this protein still possesses its secondary and tertiary structure. The charge state distribution of fully unfolded $\mathrm{CNa}$, obtained by analyzing $\mathrm{CNt}$ under denaturing conditions (data not shown), is centered around $1900 \mathrm{~m} / z$, corresponding to an average charge state of the ion that is significantly higher $(23+)$ as for partially unfolded $\mathrm{CNa}$.

Conversely, in the slightly extended $\mathrm{CNt}$ structure, already some unfolding in $\mathrm{CNb}$ occurred and the protein complex ion will dissociate via the lowest-energy route. In this particular example, it means that the small $\mathrm{CNb}$ subunit fully unfolds and dissociation of $\mathrm{CNt}$ follows the typical asymmetric route. Here, charge division is based on the accessible surface area, whereby $\mathrm{CNb}$ acquires the most.

In conclusion, although it is well established that noncovalent protein complexes dissociate via the prevalent asymmetric charge portioning mechanism expelling a highly charged smaller sub-unit, it is clear, also from the current data, that the structure of individual protein complexes may influences the dissociation behavior. By combining native tandem mass spectrometry, ion mobility mass spectrometry, and computational modeling, we reveal that for calcineurin the distinction between symmetric and asymmetric charge partitioning relies on the structure/conformation of the initial precursor ions. We propose two different explanations for this behavior. Although our data provide some evidence for heterogeneity in $\mathrm{Ca}^{2+}$ binding resulting in different $\mathrm{CNb}$ structures, we cannot exclude our second hypothesis. Here, we state that this unusual behavior likely originates from the distinctive structure of calcineurin, wherein a 22-residue helix domain of $\mathrm{CNa}$ is strongly involved in $\mathrm{CNb}$ binding. We hypothesize that this module plays an initiating role in the dissociation via the symmetric charge partitioning pathway. When more IMMS and computational modeling data become available on a variety of protein complexes, we will be able to better understand the dissociation behavior of the these complexes under tandem MS conditions, with the ultimate goal to use this sort of information for structural elucidation purposes.

\section{Acknowledgments}

The authors thank Kristina Lorenzen for helpful discussions and Arjan Barendregt and Rebecca Rose for critical reading of the manuscript and for technical support. This work was supported by The Netherlands Organization for Scientific Research (NWO) (VENI 700.58.402, EvD). B.K., I.M.B., E.vD., and A.J.R.H. thank The Netherlands Proteomics Center, embedded in the Netherlands Genomics Initiative for financial support. B.K. was supported by the Center for Biomedical Genetics.

\section{References}

1. Rumi-Masante, J., Rusinga, F.I., Lester, T.E., Dunlap, T.B., Williams, T.D., Dunker, A.K., Weis, D.D., Creamer, T.P. Structural basis for activation of calcineurin by calmodulin. J. Mol. Biol. 50(2), 307-317 (2011)

2. Rusnak, F., Mertz, P.: Calcineurin: form and function. Physiol. Rev. 80 (4), 1483-1521 (2000)

3. Wang, J.H., Desai, R.: A brain protein and its effect on the CA2+-and protein modulator-activated cyclic nucleotide phosphodiesterase. Biochem. Biophys. Res. Commun. 72(3), 926-932 (1976)

4. Stoddard, B.L., Flick, K.E.: Calcineurin-immunosuppressor complexes. Curr. Opin. Struct. Biol. 6(6), 770-775 (1996)

5. Liu, J., Farmer Jr., J.D., Lane, W.S., Friedman, J., Weissman, I., Schreiber, S.L.: Calcineurin is a common target of cyclophilin-cyclosporin A and FKBP-FK506 complexes. Cell 66(4), 807-815 (1991)

6. O’Keefe, S.J., Tamura, J., Kincaid, R.L., Tocci, M.J., O’Neill, E.A.: FK-506- and CsA-sensitive activation of the interleukin-2 promoter by calcineurin. Nature 357(6380), 692-694 (1992)

7. Clipstone, N.A., Crabtree, G.R.: Identification of calcineurin as a key signalling enzyme in T-lymphocyte activation. Nature 357(6380), 695697 (1992)

8. Li, H., Rao, A., Hogan, P.G.: Interaction of calcineurin with substrates and targeting proteins. Trends Cell Biol. 21(2), 91-103 (2011)

9. Ke, H., Huai, Q.: Structures of calcineurin and its complexes with immunophilins-immunosuppressants. Biochem. Biophys. Res. Commun. 311(4), 1095-1102 (2003)

10. Jain, J., McCaffrey, P.G., Miner, Z., Kerppola, T.K., Lambert, J.N., Verdine, G.L., Curran, T., Rao, A.: The T-cell transcription factor NFATp is a substrate for calcineurin and interacts with Fos and Jun. Nature 365(6444), 352-355 (1993)

11. Mattila, P.S., Ullman, K.S., Fiering, S., Emmel, E.A., McCutcheon, M., Crabtree, G.R., Herzenberg, L.A.: The actions of cyclosporin A and FK506 suggest a novel step in the activation of T lymphocytes. EMBO J. 9(13), 4425-4433 (1990)

12. McConnell, J.L., Wadzinski, B.E.: Targeting protein serine/threonine phosphatases for drug development. Mol. Pharmacol. 75(6), 1249-1261 (2009)

13. Klee, C.B., Crouch, T.H., Krinks, M.H.: Calcineurin: a calcium- and calmodulin-binding protein of the nervous system. Proc. Natl. Acad. Sci. U. S. A. 76(12), 6270-6273 (1979)

14. Aitken, A., Klee, C.B., Cohen, P.: The structure of the B subunit of calcineurin. Eur. J. Biochem. 139(3), 663-671 (1984)

15. Watanabe, Y., Perrino, B.A., Chang, B.H., Soderling, T.R.: Identification in the calcineurin A subunit of the domain that binds the regulatory B subunit. J. Biol. Chem. 270(1), 456-460 (1995)

16. Griffith, J.P., Kim, J.L., Kim, E.E., Sintchak, M.D., Thomson, J.A., Fitzgibbon, M.J., Fleming, M.A., Caron, P.R., Hsiao, K., Navia, M.A.: Xray structure of calcineurin inhibited by the immunophilin-immunosuppressant FKBP12-FK506 complex. Cell 82(3), 507-522 (1995)

17. Kissinger, C.R., Parge, H.E., Knighton, D.R., Lewis, C.T., Pelletier, L.A., Tempczyk, A., Kalish, V.J., Tucker, K.D., Showalter, R.E., Moomaw, E.W.: Crystal structures of human calcineurin and the human FKBP12-FK506-calcineurin complex. Nature 378(6557), 641-644 (1995) 
18. Stemmer, P.M., Klee, C.B.: Dual calcium ion regulation of calcineurin by calmodulin and calcineurin B. Biochemistry 33(22), 6859-6866 (1994)

19. Benesch, J.L.P., Ruotolo, B.T., Simmons, D.A., Robinson, C.V.: Protein complexes in the gas phase: technology for structural genomics and proteomics. Chem. Rev. 107(8), 3544-3567 (2007)

20. Benesch, J.L.P., Ruotolo, B.T., Sobott, F., Wildgoose, J., Gilbert, A., Bateman, R., Robinson, C.V.: Quadrupole-time-of-flight mass spectrometer modified for higher-energy dissociation reduces protein assemblies to peptide fragments. Anal. Chem. 81(3), 1270-1274 (2009)

21. van Duijn, E., Simmons, D.A., van den Heuvel, R.H., Bakkes, P.J., van Heerikhuizen, H., Heeren, R.M., Robinson, C.V., van der Vies, S.M., Heck, A.J.: Tandem mass spectrometry of intact GroEL-substrate complexes reveals substrate-specific conformational changes in the trans ring. J. Am. Chem. Soc. 128(14), 4694-702 (2006)

22. Zhou, M., Morgner, N., Barrera, N.P., Politis, A., Isaacson, S.C., MatakVinkovic, D., Murata, T., Bernal, R.A., Stock, D., Robinson, C.V.: Mass spectrometry of intact V-type ATPases reveals bound lipids and the effects of nucleotide binding. Science 334(6054), 380-385 (2011)

23. Light-Wahl, K.J., Schwartz, B.L., Smith, R.D.: Observation of the noncovalent quaternary association of proteins by electrospray ionization mass spectrometry. J. Am. Chem. Soc. 116, 5271-5278 (1994)

24. Sciuto, S.V., Liu, J., Konermann, L.: An electrostatic charge partitioning model for the dissociation of protein complexes in the gas phase. $J$. Am. Soc. Mass Spectrom 22, 1679-1689 (2011)

25. Pagel, K., Hyung, S.-J., Ruotolo, B.T., Robinson, C.V.: Alternate dissociation pathways identified in charge-reduced protein complex ions. Anal. Chem. 82(12), 5363-5372 (2010)

26. Li, H., Zhang, L., Rao, A., Harrison, S.C., Hogan, P.G.: Structure of calcineurin in complex with PVIVIT peptide: portrait of a low-affinity signalling interaction. J. Mol. Biol. 369(5), 1296-1306 (2007)

27. Mondragon, A., Griffith, E.C., Sun, L., Xiong, F., Armstrong, C., Liu, J.O.: Overexpression and purification of human calcineurin alpha from Escherichia coli and assessment of catalytic functions of residues surrounding the binuclear metal center. Biochemistry 36(16), 49344942 (1997)

28. Tahallah, N., Pinkse, M., Maier, C.S., Heck, A.J.: The effect of the source pressure on the abundance of ions of noncovalent protein assemblies in an electrospray ionization orthogonal time-of-flight instrument. Rapid Commun. Mass Spectrom 15(8), 596-601 (2001)

29. van den Heuvel, R.H., van Duijn, E., Mazon, H., Synowsky, S.A., Lorenzen, K., Versluis, C., Brouns, S.J., Langridge, D., van der Oost, J., Hoyes, J., Heck, A.J.: Improving the performance of a quadrupole timeof-flight instrument for macromolecular mass spectrometry. Anal. Chem. 78(21), 7473-7483 (2006)

30. Ruotolo, B.T., Benesch, J.L.P., Sandercock, A.M., Hyung, S.-J., Robinson, C.V.: Ion mobility-mass spectrometry analysis of large protein complexes. Nat. Protoc. 3(7), 1139-1152 (2008)

31. Roy, A., Kucukural, A., Zhang, Y.: I-TASSER: a unified platform for automated protein structure and function prediction. Nat. Protoc. 5(4), 725-738 (2010)

32. Zhang, H., Zhang, T., Chen, K., Shen, S., Ruan, J., Kurgan, L.: Sequence based residue depth prediction using evolutionary information and predicted secondary structure. BMC Bioinforma. 9, 388 (2008)

33. Shvartsburg, A., Jarrold, M.: An exact hard-spheres scattering model for the mobilities of polyatomic ions. Chem. Phys. Lett. 261(1/2), 86-91 (1996)

34. Uetrecht, C., Rose, R.J., van Duijn, E., Lorenzen, K., Heck, A.J.: Ion mobility mass spectrometry of proteins and protein assemblies. Chem. Soc. Rev. 39(5), 1633-1655 (2010)

35. Ruotolo, B.T., Giles, K., Campuzano, I., Sandercock, A.M., Bateman, R.H., Robinson, C.V.: Evidence for macromolecular protein rings in the absence of bulk water. Science 310(5754), 1658-1661 (2005)
36. Smith, D.P., Knapman, T.W., Campuzano, I., Malham, R.W., Berryman, J.T., Radford, S.E., Ashcroft, A.E.: Deciphering drift time measurements from traveling wave ion mobility spectrometry-mass spectrometry studies. Eur. J. Mass Spectrom 15(2), 113-130 (2009)

37. Benesch, J.L.: Collisional activation of protein complexes: picking up the pieces. J. Am. Soc. Mass Spectrom. 20(3), 341-348 (2009)

38. Lorenzen, K., Vannini, A., Cramer, P., Heck, A.J.: Structural biology of RNA polymerase III: mass spectrometry elucidates subcomplex architecture. Structure 15(10), 1237-1245 (2007)

39. Scarff, C.A., Thalassinos, K., Hilton, G.R., Scrivens, J.H.: Travelling wave ion mobility mass spectrometry studies of protein structure: biological significance and comparison with X-ray crystallography and nuclear magnetic resonance spectroscopy measurements. Rapid Commun. Mass Spectrom. 22(20), 3297-3304 (2008)

40. Benesch, J.L., Ruotolo, B.T.: Mass spectrometry: come of age for structural and dynamical biology. Curr. Opin. Struct. Biol. 21(5), 641649 (2011)

41. Hall, Z., Politis, A., Bush, M.F., Smith, L.J., Robinson, C.V.: Charge state-dependent compaction and dissociation of protein complexes: insights from ion mobility and molecular dynamics. J. Am. Chem. Soc. 134(7), 3429-3438 (2012)

42. Bleiholder, C., Wyttenbach, T., Bowers, M.T.: A novel projection approximation algorithm for the fast and accurate computation of molecular collision cross sections (I) Method. Int. J. Mass Spectrom. 308, 1-10 (2011)

43. Uetrecht, C., Heck, A.J.: Modern biomolecular mass spectrometry and its role in studying virus structure, dynamics, and assembly. Angew. Chem. 33, 6247-6251 (2011)

44. Beardsley, R.L., Jones, C.M., Galhena, A.S., Wysocki, V.H.: Noncovalent protein tetramers and pentamers with " $n$ " charges yield monomers with $\mathrm{n} / 4$ and n/5 charges. Anal. Chem. 81(4), 1347-1356 (2009)

45. Jurchen, J.C., Williams, E.R.: Origin of asymmetric charge partitioning in the dissociation of gas-phase protein homodimers. J. Am. Chem. Soc. 125(9), 2817-2826 (2003)

46. Madsen, J.A., Brodbelt, J.S.: Asymmetric charge partitioning upon dissociation of DNA duplexes. J. Am. Soc. Mass Spectrom. 21(7), 1144-1150 (2010)

47. Jones, C.M., Beardsley, R.L., Galhena, A.S., Dagan, S., Cheng, G., Wysocki, V.H.: Symmetrical gas-phase dissociation of noncovalent protein complexes via surface collisions. J. Am. Chem. Soc. 128(47), 15044-15045 (2006)

48. Wysocki, V.H., Jones, C.M., Galhena, A.S., Blackwell, A.E.: Surfaceinduced dissociation shows potential to be more informative than collision-induced dissociation for structural studies of large systems. $J$. Am. Soc. Mass Spectrom. 19(7), 903-913 (2008)

49. Wanasundara, S.N., Thachuk, M.: Theoretical investigations of the dissociation of charged protein complexes in the gas phase. J. Am. Soc. Mass Spectrom. 18(12), 2242-2253 (2007)

50. Kang, Y., Douglas, D.J.: Gas-phase ions of human hemoglobin A, F, and S. J. Am. Soc. Mass Spectrom. 22(7), 1187-1196 (2011)

51. Lorenzen, K., van Duijn, E. (2010). Native mass spectrometry as a tool in structural biology. Curr. Protoc. Prot. Sci. 17, Unit17.12

52. Jurchen, J.C., Garcia, D.E., Williams, E.R.: Gas-phase dissociation pathways of multiply charged peptide clusters. J. Am. Chem. Soc. 14 (12), 1373-1386 (2003)

53. Jurchen, J.C., Garcia, D.E., Williams, E.R.: Further studies on the origins of asymmetric charge partitioning in protein homodimers. $J$. Am. Soc. Mass Spectrom. 15(10), 1408-1415 (2004)

54. Yang, S.A., Klee, C.B.: Low affinity $\mathrm{Ca} 2+$-binding sites of calcineurin B mediate conformational changes in calcineurin A. Biochemistry 39 (51), 16147-16154 (2000) 